\section{PL.08 EVALUATION OF A NEW FORMATIVE ASSESSMENT TOOL FOR VACUUM DELIVERY}

doi:10.1136/archdischild-2013-303966.193

J Farey, V Stern, B Strachan, R Bahl. St Michael's Hospital, Bristol, UK

Introduction Instrumental deliveries account for 10-13\% of births in the UK. Achieving competence in vacuum delivery is a mandatory requirement of the RCOG core curriculum. OSATS are the current tool used for formative and summative work based assessment of practical skills. Performance of each subtask is classified as competent or 'needs help'. The level of expertise for each subtask is not individually defined limiting the detail of the feedback for formative assessment.

Aim To ascertain the inter-rater reliability of a new formative assessment tool where the construct validity has already been established.

Methods 22 videos of vacuum delivery conducted by obstetricians of varying grade were reviewed independently by two assessors. The formative assessment tool was completed for each video recording. There were a total of 12 subtasks, each scored between 1 and 5.

Results The scores for each video ranged from 39 to 60 . The total scores for each video recording were compared using the Cohen Kappa co-efficient, with a resulting co-efficient of 0.62 (SE 0.08, 95\% CI $0.44-0.78$ ) indicating strong reliability.

Discussion Reduction in training hours has made experiential learning more challenging and therefore effective training and assessment tools are increasingly important. This new tool aims to give more detailed feedback and provide a thorough assessment of the necessary steps in performing a competent vacuum delivery.

We believe this tool shows promise as an alternative to the current standard of assessment.

\section{PL.09 SECOND-STAGE CAESAREAN SECTIONS: HOW TO DELIVER AND HOW TO TRAIN?}

doi:10.1136/archdischild-2013-303966.194

N Vousden, A Briley, G Tydeman, Z Hamakarim, P Seed, AH Shennan. Division of Womens Health, Kings College London, Academic Womens Health Centre, Kings Health Partners, London, UK

Background Approximately 8000 full dilatation caesarean sections (CS) are performed in the UK each year ${ }^{1}$. Delivery can be technically challenging and associated with greater maternal and fetal morbidity ${ }^{1}$. There are no national guidelines. The aim of this study is to determine expert consensus on the most important techniques for safe delivery and to validate a second-stage CS simulation device ('Desperate Debra').

Method Results of questionnaires were independently analysed by two people. Specialists performed three deliveries with different fetal positions and degrees of impaction on a second-stage CS simulator and completed visual analogue scores (VAS).

Results Responses were received from 47 specialists ( $>7$ years experience) with a mean 18 years of labour-ward experience. The five most reported important techniques for safe delivery were 1) high uterine incision $(\mathrm{n}=28), 2)$ assistance to push fetal head transvaginally $(n=25), 3)$ correct flexion of fetal head $(n=20)$, 4) determine fetal position prior to starting $(n=17)$ and 5$)$ disempaction of the fetal head in the caudal direction prior to elevation $(n=13)$. The training scenarios were performed by 30 specialists (mean 7 years experience). The degrees of impaction (1.light, 2.moderate, 3.severe) correlated with perceived difficulty of delivery (mean VAS 29/100, $42 / 100$ and $88 / 100$ respectively; $1-2$ vs. 3 linear regression $p<0.001$ ). Delivery was successful in $100 \%, 90 \%$ and $61 \%$ respectively (1-2 vs. 3 binomial regression $\mathrm{p}<0.05) .87 \%$ of specialists found it realistic and $93 \%$ useful.
Conclusions The simulator device is a valuable training resource. It should be used in conjugation with currently accepted techniques while further research on optimal management is awaited.

\section{REFERENCE}

1. Loudon JAZ, Groom KM, Hinkson L, Harrington D \& Paterson-Brown S. Changing trends in operative delivery performed at full dilatation over a 10-year period. Journal of Obstetrics and Gynaecology 2010;30:370-375.

\section{PL.10 CAN THE INSTITUTE FOR HEALTHCARE IMPROVEMENT "MODEL FOR IMPROVEMENT" REDUCE OBSTETRIC ANAL SPHINCTER INJURIES (OASIS)?}

doi:10.1136/archdischild-2013-303966.195

W Maina, J Mansfield, C Jones, J Tinsey, M Cameron. Norfolk and Norwich University Hospital, Norwich, UK

Introduction Dr Foster reported obstetric anal sphincter injuries (OASIS) incidence in Norwich as $3.72 \%$ for spontaneous vaginal deliveries and $10.76 \%$ for instrumental deliveries against expected incidence of $3.6 \%$ and $7.6 \%$ respectively. The Norfolk and Norwich Improving Patient Safety (NNIPS) programme is modelled on Leading Improvement in Patient Safety (LIPS) programme (NHS Institute for Innovation and Improvement) which uses the Institute for Healthcare Improvement (IHI) Model for Improvement.

Setting UK university teaching hospital delivering 6000 babies annually.

Aim To reduce OASIS by $20 \%$ within nine months using IHI methodology.

Methods The nine month NNIPS programme involved four one day workshops and four mentoring sessions to equip teams with improvement techniques. Methodology employed in this project included:
1. Ishikawa diagram
2. Process mapping
3. Model for Improvement: Plan-Do-Study-Act (PDSA)
4. Statistical process control (SPC) charts for measurement

Results

1. Ishikawa diagram - identified two issues: diagnosis and prevention.

2. Process mapping identified up to four rectal examinations were required before OASIS diagnosis and no standardised technique of perineal examination. Redundant steps were removed and diagnosis was standardised in PDSA 1 and 2.

3. Prevention: PDSA 3 involved the development of an agreed method of managing the perineum in second stage called "SLOWER."PDSA 4 involved group reflection with midwives.

4. SPC Charts: failed to demonstrate any change in OASIS.

Conclusion We were unable to demonstrate any statistical reduction in OASIS although processes improved over the time frame.

\section{PL.11 WITHDRAWN BY AUTHOR}

\section{PL.12 SHORT TERM CLINICAL OUTCOMES OF NEONATES WITH SEVERE PERINATAL ACIDOSIS: A PROSPECTIVE STUDY}

doi:10.1136/archdischild-2013-303966.196

'A Marthandan, 'S Ramaiah, ${ }^{2} \mathrm{D}$ Shah, 'R Roy, 'P Clarke. 'Neonatal Unit, Norfolk and Norwich University Hospitaly Hospital, Norwich, UK; ${ }^{2}$ Neonatal Unit, Royal London Hospital, London, UK

Background Therapeutic hypothermia improves outcomes for asphyxiated infants, however not all infants born with severe acidosis are assessed as eligible for cooling and few data describe shortterm clinical outcomes for the whole cohort of babies with severe perinatal acidosis. 